UDC 517.956 .25

\title{
BOUNDEDNESS OF SOLUTIONS TO ANISOTROPIC SECOND ORDER ELLIPTIC EQUATIONS IN UNBOUNDED DOMAINS
}

\section{L.M. KOZHEVNIKOVA, A.A. KHADZHI}

\begin{abstract}
In the paper we study a class of anisotropic second order elliptic equations represented by the model equation

$$
\sum_{\alpha=1}^{n}\left(\left|u_{x_{\alpha}}\right|^{p_{\alpha}-2} u_{x_{\alpha}}\right)_{x_{\alpha}}=\sum_{\alpha=1}^{n}\left(\Phi_{\alpha}(\mathbf{x})\right)_{x_{\alpha}}, \quad p_{n} \geqslant \ldots \geqslant p_{1}>1 .
$$

We prove the boundedness of solutions to the homogeneous Dirichlet problem in unbounded domains located along one of the coordinate axes. We also establish an estimate for the solutions to the considered equations with a compactly supported right hand side that ensures a power decay of the solutions at infinity.
\end{abstract}

Keywords: Dirichlet problem, anisotropic elliptic equation, unbounded domain, boundedness of solutions, decay of solution.

\section{Mathematics Subject Classification: $35 \mathrm{~J} 62$}

\section{INTRODUCTION}

Let $\Omega$ be an arbitrary unbounded domain in the space $\mathbb{R}_{n}=\left\{\mathbf{x}=\left(x_{1}, x_{2}, \ldots, x_{n}\right)\right\}, \Omega \subseteq \mathbb{R}_{n}$, $n \geqslant 2$. We consider the Dirichlet problem for an anisotropic quasilinear second order elliptic equation

$$
\begin{gathered}
\sum_{\alpha=1}^{n}\left(a_{\alpha}(\mathbf{x}, \nabla u)\right)_{x_{\alpha}}=\sum_{\alpha=1}^{n}\left(\Phi_{\alpha}(\mathbf{x})\right)_{x_{\alpha}}, \quad \mathbf{x} \in \Omega ; \\
\left.u\right|_{\partial \Omega}=0 .
\end{gathered}
$$

We assume that functions $a_{\alpha}(\mathbf{x}, \xi), \alpha=\overline{1, n}$, are measurable w.r.t. $\mathbf{x} \in \Omega$ for $\xi \in \mathbb{R}_{n}$ and continuous w.r.t. $\xi \in \mathbb{R}_{n}$ for a.e. $\mathbf{x} \in \Omega$. Let $\mathbf{p}=\left(p_{1}, p_{2}, \ldots, p_{n}\right)$; we suppose that $1<p_{1} \leqslant p_{2} \leqslant \ldots \leqslant p_{n}$ and there exists positive numbers $\bar{a}$, $\widehat{a}$ such that for each $\xi, \eta \in \mathbb{R}_{n}$ and a.e. $\mathbf{x} \in \Omega$ the conditions

$$
\begin{gathered}
\sum_{\alpha=1}^{n}\left(a_{\alpha}(\mathbf{x}, \xi)-a_{\alpha}(\mathbf{x}, \eta)\right)\left(\xi_{\alpha}-\eta_{\alpha}\right) \geqslant \bar{a} \sum_{\alpha=1}^{n}\left|\xi_{\alpha}-\eta_{\alpha}\right|^{p_{\alpha}} \\
\left|a_{\alpha}(\mathbf{x}, \xi)-a_{\alpha}(\mathbf{x}, \eta)\right| \leqslant \widehat{a}\left|\xi_{\alpha}-\eta_{\alpha}\right|\left(\left|\xi_{\alpha}\right|+\left|\eta_{\alpha}\right|\right)^{p_{\alpha}-2}, \quad \alpha=1,2, \ldots, n ; \\
a_{\alpha}(\mathbf{x}, \mathbf{0})=0, \quad \alpha=1,2, \ldots, n,
\end{gathered}
$$

are satisfied.

L.M. Kozhevnikova, A.A. Khadzhi, Boundedness of SOlutions to Anisotropic SeCond ORder ELLIPTIC EQUATIONS IN UNBOUNDED DOMAINS.

(c) Kozhevnikova L.M., Khadzhi A.A. 2014.

The work is supported by RFBR (grant no. 13-01-00081-a).

Submitted November 5, 2013. 
I.M. Kolodii [1] established the boundedness of solutions to some class of anisotropic elliptic equations in bounded domains. At that, the boundedness of the domain was essential in his proof. The main result of the present paper is the proof of the boundedness for the generalized solutions to problem (1), (2) in unbounded domains $\Omega$.

We suppose that $\Phi_{\alpha}(\mathbf{x}) \in L_{p_{\alpha} /\left(p_{\alpha}-1\right)}(\Omega), \alpha=1,2, \ldots, n$. The generalized solution to problem (1), (2) is treated in a "narrow" sense, i.e., as an element in appropriate anisotropic Sobolev space $H_{\mathbf{p}}^{1}(\Omega)$ introduced as the completion of the space $C_{0}^{\infty}(\Omega)$ w.r.t. the norm $\|v\|_{H_{\mathbf{p}}^{1}(\Omega)}=$ $\sum_{\alpha=1}^{n}\left\|v_{x_{\alpha}}\right\|_{L_{p_{\alpha}}(\Omega)}$ (the definition of the latter is given in Section 2).

In the paper we consider domains located along a selected axis $O x_{s}, s=\overline{1, n}$ (domain $\Omega$ lies in the half-space $x_{s}>0$ and the cross-section $\gamma_{r}=\left\{\mathbf{x} \in \Omega \mid x_{s}=r\right\}$ is non-empty for each $r>0)$.

We introduce the notation: $\Omega_{a}^{b}=\left\{\mathbf{x} \in \Omega \mid a<x_{s}<b\right\}$, values $a=0, b=\infty$ can be omitted. Let $P=n\left(-1+\sum_{\alpha=1}^{n} 1 / p_{\alpha}\right)^{-1}, M=p_{s}\left(P-p_{s}\right)^{-1}, K=\sum_{\alpha=1}^{n} 1 / p_{\alpha}\left(-1+\sum_{\alpha=1}^{n} 1 / p_{\alpha}\right)^{-1}$.

Theorem 1. Let $u(\mathbf{x})$ be a generalized solution to problem (1), (2) with

$$
\operatorname{supp} \Phi_{\alpha} \subset \Omega^{R_{0}}, \quad R_{0}>0, \quad \alpha=1,2, \ldots, n,
$$

and conditions (3) - (5) are satisfied as well as

$$
1<\sum_{\alpha=1}^{n} \frac{1}{p_{\alpha}}<1+\frac{n}{p_{s}} .
$$

Then for $R \geqslant 2 R_{0} / \varepsilon, \varepsilon \in(0,1)$, the inequality

$$
\text { vrai } \max _{\Omega_{\varepsilon R}^{R}}|u(\mathbf{x})| \leqslant \frac{\widetilde{C}}{R^{M}}
$$

holds true, where $\widetilde{C}$ is a positive constant depending on $p_{\alpha}, n, \bar{a}, \widehat{a},\left\|\Phi_{\alpha}\right\|_{p_{\alpha} /\left(p_{\alpha}-1\right)}$.

Example 1. Let $p_{\alpha}=p, \alpha=1,2, \ldots, n$. In the ball $B_{1}$ of radius 1 centered at the origin we consider the function $u(\mathbf{x})=\ln r, r=|\mathbf{x}|$. It is an unbounded solution to equation (1) with $\Phi_{\alpha}(\mathbf{x})=\left|u_{x_{\alpha}}\right|^{p-2} u_{x_{\alpha}} \in L_{p /(p-1)}, p<n$. Thus, even in the isotropic case the belongings $\Phi_{\alpha}(\mathbf{x}) \in L_{p /(p-1)}, \alpha=1,2, \ldots, n$, are insufficient for the boundedness of solutions.

In the next theorem we prove the boundedness of a solution to problem (1), (2) ( $\Omega$ is unbounded) in $\Omega^{R_{1}}$ for arbitrary $R_{1}>0$ under the assumption of higher summability of functions $\Phi_{\alpha}(\mathbf{x})$ (in particular, they can be bounded).

Theorem 2. Let $u(\mathbf{x})$ be a generalized solution to problem (1), (2) with functions $\Phi_{\alpha}(\mathbf{x})$ such that for each $r>0$

$$
\begin{gathered}
\Phi_{\alpha}(\mathbf{x}) \in L_{k_{\alpha}}\left(\Omega^{r}\right), \quad k_{\alpha}=\frac{p_{\alpha} l}{\left(p_{\alpha}-1\right)(l-1)}, \quad \alpha=1,2, \ldots, n, \\
1 \leqslant l<\min \left(K, \frac{P}{p_{s}}\right),
\end{gathered}
$$

and conditions (3)-(5) are obeyed with exponents $p_{\alpha}$ such that

$$
1<\sum_{\alpha=1}^{n} \frac{1}{p_{\alpha}}<1+\min \left\{\frac{n}{l p_{s}}, \frac{1}{l-1}\right\} .
$$


Then for each $R_{1}>0$ the estimate

$$
\text { vrai } \max _{\Omega^{R_{1}}}|u(\mathbf{x})| \leqslant \bar{C}
$$

holds true, where $\bar{C}$ is a positive constant depending on $p_{\alpha}, n, l, \bar{a}, \widehat{a}, R_{1}$, mes $\Omega^{2 R_{1}},\left\|\Phi_{\alpha}\right\|_{k_{\alpha}, \Omega^{2 R_{1}}}$.

Example 2. Let $p_{1}<p_{n}<p_{1} \sum_{\alpha=1}^{n} 1 / p_{\alpha}$. In the ball $B_{1}$ we consider the function $u(\mathbf{x})=r^{-A}$, $r=|\mathbf{x}|, \quad A=\frac{n}{p_{1} \sum_{\alpha=1}^{n} 1 / p_{\alpha}}-1>0$. It is an unbounded solution to equation (1) with functions $\Phi_{s}(\mathbf{x})=\left|u_{x_{s}}\right|^{p_{s}-2} u_{x_{s}}, s=1,2, \ldots, n$. It is easy to check that functions $\Phi_{s}(\mathbf{x})$ are $r_{s}$-power integrable functions in the ball $B_{1}$, and this exponent is less than $\frac{n}{(A+1)\left(p_{s}-1\right)}$, while the exponents $k_{s}$ in Theorem 2 are greater than $\frac{p_{s}}{p_{s}-1} \sum_{\alpha=1}^{n} 1 / p_{\alpha}$. Since $r_{s}<\frac{p_{1} \sum_{\alpha=1}^{n} 1 / p_{\alpha}}{\left(p_{s}-1\right)} \leqslant k_{s}, s=1,2, \ldots, n$, we can state that the lower bound of the integrability exponents $k_{s}$ for functions $\Phi_{s}$ is close to the lowest possible.

In [2] the authors obtained the estimates for the decay at infinity of solution to anisotropic elliptic equations subject to the geometry of unbounded domain $\Omega$ located along a selected axis; this was done for bounded solutions. However, the boundedness left unproven. The main aim of the present paper is the proof of global boundedness for a generalize solutions to problem (1), (2). It is sure that for an isotropic equations one can omit the restriction for the class of considered domains, but in the case of anisotropic equations it leads one to substantial technical difficulties in the proof of estimate (8). Estimate (12) can be obtained for arbitrary unbounded domains with a non-compact boundary. But here we provide its proof for domains located along a selected axis for the consistency with estimate (8). A corollary of Theorems 1, 2 is

Theorem 3. Suppose that conditions (3)-(5), (11) hold true. Then a generalized solution to problem (1), (2) $u(\mathbf{x})$ with functions $\Phi_{\alpha}(\mathbf{x}), \alpha=\overline{1, n}$, satisfying (6), (9), satisfies the estimate

$$
\sup _{\Omega}|u| \leqslant C
$$

where $C$ is a constant depending on $p_{\alpha}, n, \bar{a}, \widehat{a},\left\|\Phi_{\alpha}\right\|_{k_{\alpha}}, R_{0}$, mes $\Omega^{4 R_{0}}, l$.

\section{Auxiliary Statements}

We denote by $\|\cdot\|_{p}$ the norm in the space $L_{p}(\Omega)$. Let us provide an embedding theorem for the anisotropic Sobolev space implying that $\|\cdot\|_{H_{\mathbf{p}}^{1}(\Omega)}$ is a norm.

Lemma 1. Let $u(\mathbf{x}) \in \stackrel{\circ}{H}{ }_{\mathbf{p}}^{1}(\Omega)$ and

$$
\sum_{\alpha=1}^{n} 1 / p_{\alpha}>1
$$

Then $u(\mathbf{x}) \in L_{P}(\Omega)$, where $P=n\left(-1+\sum_{\alpha=1}^{n} 1 / p_{\alpha}\right)^{-1}$, and

$$
\|u\|_{P} \leqslant A_{1} \sum_{\alpha=1}^{n}\left\|u_{x_{\alpha}}\right\|_{p_{\alpha}}
$$

Here $A_{1}$ is a constant depending on $p_{\alpha}, n$ (see [3, [4]). 
Definition 1. A generalized solution to problem (1), (2) with $\Phi_{\alpha}(\mathbf{x}) \in L_{p_{\alpha} /\left(p_{\alpha}-1\right)}(\Omega)$, $\alpha=1,2, \ldots, n$, is a function $u(\mathbf{x}) \in \stackrel{\circ}{H}_{\mathbf{p}}^{1}(\Omega)$ obeying the integral identity

$$
\int_{\Omega} L(u, v) d \mathbf{x} \equiv \int_{\Omega} \sum_{\alpha=1}^{n}\left(a_{\alpha}(\mathbf{x}, \nabla u)-\Phi_{\alpha}\right) v_{x_{\alpha}} d \mathbf{x}=0
$$

for each function $v(\mathbf{x}) \in \stackrel{\circ}{H}_{\mathbf{p}}^{1}(\Omega)$.

Theorem 4. Suppose that conditions (3)-(5) are satisfied. Then there exists the unique generalized solution $u(\mathbf{x})$ to problem (1), (2) with functions $\Phi_{\alpha}(\mathbf{x}) \in L_{p_{\alpha} /\left(p_{\alpha}-1\right)}(\Omega), \alpha=1,2, \ldots, n$, and the estimate

$$
\sum_{\alpha=1}^{n}\left\|u_{x_{\alpha}}\right\|_{p_{\alpha}}^{p_{\alpha}} \leqslant A_{2} \sum_{\alpha=1}^{n}\left\|\Phi_{\alpha}\right\|_{p_{\alpha} /\left(p_{\alpha}-1\right)}^{p_{\alpha} /\left(p_{\alpha}-1\right)}
$$

is valid, where $A_{2}$ is constant depending on $\bar{a}, \widehat{a}, p_{\alpha}$.

The proof of the existence is made by Galerkin's approximations.

Lemma 2. As $0 \leqslant a<b$, a function $u(\mathbf{x}) \in \stackrel{\circ}{H_{\mathbf{p}}^{1}}(\Omega)$ satisfies the inequality

$$
\frac{1}{b}\|u\|_{p_{s}, \Omega_{a}^{b}} \leqslant \frac{p_{s}}{p_{s}-1}\left\|u_{x_{s}}\right\|_{p_{s}}
$$

(see [5, Ineq. (73)]).

Lemma 3. Let $u(\mathbf{x}) \in \stackrel{\circ}{H_{\mathbf{p}}^{1}}(D)$ and

$$
\sum_{\alpha=1}^{n} \int_{D}|u|^{q_{\alpha}}\left|u_{x_{\alpha}}\right|^{p_{\alpha}} d \mathbf{x}<\infty, \quad q_{\alpha} \geqslant 0, \quad p_{\alpha} \geqslant 1, \quad \alpha=1,2, \ldots, n .
$$

If condition (13) is satisfied, then $u(\mathbf{x}) \in L_{Q}(D)$ as $Q=\sum_{\alpha=1}^{n}\left(1+q_{\alpha} / p_{\alpha}\right)\left(-1+\sum_{\alpha=1}^{n} 1 / p_{\alpha}\right)^{-1}$, and the estimate

$$
\|u\|_{Q, D} \leqslant A_{3}\left(\sum_{\alpha=1}^{n} \int_{D}|u|^{q_{\alpha}}\left|u_{x_{\alpha}}\right|^{p_{\alpha}} d \mathbf{x}\right)^{K / Q}
$$

is valid, where $K=\sum_{\alpha=1}^{n} 1 / p_{\alpha}\left(-1+\sum_{\alpha=1}^{n} 1 / p_{\alpha}\right)^{-1}, A_{3}$ is a constant depending on $n, q_{\alpha}, p_{\alpha}$ (see [3], [6], [7]).

Remark. It was shown by V.S. Klimov in [8] that inequality 18 is valid also for functions "vanishing on a rather massive subset of $\bar{\Omega}$ ". In particular, it is true as $D=\Omega^{r}, r>0$, for functions $u(\mathbf{x}) \in \stackrel{\circ}{H}_{\mathbf{p}}^{1}(\Omega)$.

\section{Proof of Theorems 1,2}

The proofs of Theorems 1 and 2 are based on the iterative method suggested by Yu. Moser [9] and widely used in works by S.N. Kruzhkov [10], [4], D. Serrin [11], I.M. Kolodii [1].

We let $\bar{u}(\mathbf{x})=|u(\mathbf{x})|+\chi, \chi \geqslant 0$, and $\left|u_{x_{\alpha}}\right|=\left|\bar{u}_{x_{\alpha}}\right|$. For fixed numbers $q \geqslant 1$ and $\mu>\chi$ we define the functions

$$
\begin{gathered}
F(\bar{u})= \begin{cases}\bar{u}^{q} & \text { if } \quad \chi \leqslant \bar{u} \leqslant \mu, \\
q \mu^{q-1} \bar{u}-(q-1) \mu^{q} & \text { if } \mu<\bar{u},\end{cases} \\
G(u)=\left\{F(\bar{u}) F^{\prime}(\bar{u})^{p_{s}-1}-\chi^{q p_{s}-p_{s}+1} q^{p_{s}-1}\right\} \operatorname{sign} u, \quad-\infty<u<\infty .
\end{gathered}
$$


A.e. on the set $\{\mathbf{x}: \bar{u} \neq \mu\}$ we have

$$
0 \leqslant G^{\prime}(u)= \begin{cases}\frac{p_{s} q-p_{s}+1}{q} F^{\prime}(\bar{u})^{p_{s}} & \text { if } \quad \bar{u} \leqslant \mu \\ F^{\prime}(\bar{u})^{p_{s}} & \text { if } \quad \mu<\bar{u}\end{cases}
$$

The inequalities

$$
\begin{gathered}
p_{s} F^{\prime}(\bar{u})^{p_{s}} \geqslant G^{\prime}(u) \geqslant F^{\prime}(\bar{u})^{p_{s}}, \quad|G(u)| \leqslant F(\bar{u}) F^{\prime}(\bar{u})^{p_{s}-1} \\
F(\bar{u}) \leqslant \bar{u}^{q}, \quad F^{\prime}(\bar{u}) \leqslant q \bar{u}^{q-1}
\end{gathered}
$$

hold true.

Proof of Theorem 1. Let $\eta\left(x_{s}\right)$ be a non-negative Lipschitz function with the support in $[\bar{\rho}-$ $\bar{\sigma}, \widehat{\rho}+\widehat{\sigma}] \subset[\varepsilon R / 2,2 R], \varepsilon \in(0,1)$, such that

$$
\eta\left(x_{s}\right)= \begin{cases}1, & x_{s} \in[\bar{\rho}, \widehat{\rho}] \\ 0, & x_{s} \notin(\bar{\rho}-\bar{\sigma}, \widehat{\rho}+\widehat{\sigma}) \\ \text { linear, } & x_{s} \in[\bar{\rho}-\bar{\sigma}, \bar{\rho}) \cup(\widehat{\rho}, \widehat{\rho}+\widehat{\sigma}]\end{cases}
$$

We let $v(\mathbf{x})=\eta^{p_{s}} G(u) \in \stackrel{\circ}{H}_{\mathbf{p}}^{1}(\Omega), \chi=0$. A.e. on the set $\{\mathbf{x}:|u| \neq \mu\}$ we have

$$
v_{x_{\alpha}}=\eta^{p_{s}} G^{\prime}(u) u_{x_{\alpha}}+p_{s} \eta^{p_{s}-1} G(u) \eta_{x_{\alpha}}, \quad \alpha=1,2, \ldots, n .
$$

Employing (19), (6), we find

$$
\begin{aligned}
L(u, v) & =\sum_{\alpha=1}^{n}\left(a_{\alpha}(\mathbf{x}, \nabla u)-\Phi_{\alpha}\right)\left(p_{s} \eta^{p_{s}-1} G(u) \eta_{x_{\alpha}}+\eta^{p_{s}} G^{\prime}(u) u_{x_{\alpha}}\right) \\
& \geqslant \eta^{p_{s}} F^{\prime}(|u|)^{p_{s}} \sum_{\alpha=1}^{n} a_{\alpha}(\mathbf{x}, \nabla u) u_{x_{\alpha}}-p_{s} \eta^{p_{s}-1}\left|\eta_{x_{s}}\right| F(|u|) F^{\prime}(|u|)^{p_{s}-1}\left|a_{s}(\mathbf{x}, \nabla u)\right| .
\end{aligned}
$$

By conditions (3)-(5), we obtain

$$
L(u, v) \geqslant \bar{a} \eta^{p_{s}} F^{\prime}(|u|)^{p_{s}} \sum_{\alpha=1}^{n}\left|u_{x_{\alpha}}\right|^{p_{\alpha}}-\widehat{a} p_{s} \eta^{p_{s}-1} F(|u|) F^{\prime}(|u|)^{p_{s}-1}\left|\eta_{x_{s}}\right|\left|u_{x_{s}}\right|^{p_{s}-1} .
$$

Integrating (21) over $\mathbf{x} \in \Omega$ and taking into consideration definition (15), we get

$$
\int_{\Omega} \eta^{p_{s}} F^{\prime}(|u|)^{p_{s}} \sum_{\alpha=1}^{n}\left|u_{x_{\alpha}}\right|^{p_{\alpha}} d \mathbf{x} \leqslant C_{1} \int_{\Omega} F(|u|) F^{\prime}(|u|)^{p_{s}-1} \eta^{p_{s}-1}\left|\eta_{x_{s}}\right|\left|u_{x_{s}}\right|^{p_{s}-1} d \mathbf{x} .
$$

Young inequality implies

$$
\int_{\Omega} \eta^{p_{s}} F^{\prime}(|u|)^{p_{s}} \sum_{\alpha=1}^{n}\left|u_{x_{\alpha}}\right|^{p_{\alpha}} d \mathbf{x} \leqslant \frac{1}{2} \int_{\Omega} \eta^{p_{s}} F^{\prime}(|u|)^{p_{s}}\left|u_{x_{s}}\right|^{p_{s}} d \mathbf{x}+C_{2} \int_{\Omega} F(|u|)^{p_{s}}\left|\eta_{x_{s}}\right|^{p_{s}} d \mathbf{x} .
$$

It follows from 20 that

$$
\begin{aligned}
q^{p_{s}} \int_{\{x \in \Omega:|u| \leqslant \mu\}} \eta^{p_{s}}|u|^{(q-1) p_{s}} \sum_{\alpha=1}^{n}\left|u_{x_{\alpha}}\right|^{p_{\alpha}} d \mathbf{x} & \leqslant \int_{\Omega} \eta^{p_{s}} F^{\prime}(|u|)^{p_{s}} \sum_{\alpha=1}^{n}\left|u_{x_{\alpha}}\right|^{p_{\alpha}} d \mathbf{x} \\
& \leqslant C_{3} \int_{\Omega}|u|^{q p_{s}}\left|\eta_{x_{s}}\right|^{p_{s}} d \mathbf{x} .
\end{aligned}
$$


Assume that the right hand side of $(22)$ is finite. We let $\mu$ tend to infinity in the left hand side of (22) and apply Fatou lemma

$$
\int_{\Omega} \eta^{p_{s}}|u|^{p_{s}(q-1)} \sum_{\alpha=1}^{n}\left|u_{x_{\alpha}}\right|^{p_{\alpha}} d \mathbf{x} \leqslant \frac{C_{3}}{q^{p_{s}}} \int_{\Omega}|u|^{q p_{s}}\left|\eta_{x_{s}}\right|^{p_{s}} d \mathbf{x} .
$$

We obtain a chain of inequalities

$$
\begin{aligned}
& \sum_{\alpha \neq s} \int_{\Omega}\left(|u| \eta^{p_{s} / p_{1}}\right)^{p_{s}(q-1)}\left|\left(u \eta^{p_{s} / p_{1}}\right)_{x_{\alpha}}\right|^{p_{\alpha}} d \mathbf{x}+\int_{\Omega}\left(|u| \eta^{p_{s} / p_{1}}\right)^{p_{s}(q-1)}\left|\left(u \eta^{p_{s} / p_{1}}\right)_{x_{s}}\right|^{p_{s}} d \mathbf{x} \\
& =\sum_{\alpha \neq s} \int_{\Omega}|u|^{p_{s}(q-1)}\left|u_{x_{\alpha}}\right|^{p_{\alpha}} \eta^{p_{s} / p_{1}\left[p_{s}(q-1)+p_{\alpha}\right]} d \mathbf{x} \\
& +\int_{\Omega} \eta^{p_{s}^{2}(q-1) / p_{1}}|u|^{p_{s}(q-1)}\left|u_{x_{s}} \eta^{p_{s} / p_{1}}+\frac{p_{s}}{p_{1}} u \eta^{p_{s} / p_{1}-1} \eta_{x_{s}}\right|^{p_{s}} d \mathbf{x} \\
& \leqslant \sum_{\alpha \neq s} \int_{\Omega}|u|^{p_{s}(q-1)}\left|u_{x_{\alpha}}\right|^{p_{\alpha}} \eta^{p_{s} / p_{1}\left[p_{s}(q-1)+p_{\alpha}\right]} d \mathbf{x} \\
& +C_{4} \int_{\Omega} \eta^{p_{s}^{2} q / p_{1}}|u|^{p_{s}(q-1)}\left|u_{x_{s}}\right|^{p_{s}} d \mathbf{x}+C_{4} \int_{\Omega} \eta^{p_{s}\left[q p_{s} / p_{1}-1\right]}\left|\eta_{x_{s}}\right|^{p_{s}}|u|^{p_{s} q} d \mathbf{x} \\
& \leqslant C_{5} \sum_{\alpha=1}^{n} \int_{\Omega}|u|^{p_{s}(q-1)}\left|u_{x_{\alpha}}\right|^{p_{\alpha}} \eta^{p_{s}\left[p_{s}(q-1)+p_{\alpha}\right] / p_{1}} d \mathbf{x}+C_{4} \int_{\Omega}|u|^{p_{s} q} \eta^{p_{s}\left[q p_{s}-p_{1}\right] / p_{1}}\left|\eta_{x_{s}}\right|^{p_{s}} d \mathbf{x} .
\end{aligned}
$$

Since $0 \leqslant \eta\left(x_{s}\right) \leqslant 1$, we apply 23 to get

$$
\begin{aligned}
& \sum_{\alpha=1}^{n} \int_{\Omega}\left|u \eta^{p_{s} / p_{1}}\right|^{p_{s}(q-1)}\left|\left(u \eta^{p_{s} / p_{1}}\right)_{x_{\alpha}}\right|^{p_{\alpha}} d \mathbf{x} \\
& \leqslant C_{5} \sum_{\alpha=1}^{n} \int_{\Omega}|u|^{p_{s}(q-1)}\left|u_{x_{\alpha}}\right|^{p_{\alpha}} \eta^{p_{s}} d \mathbf{x}+C_{4} \int_{\Omega}|u|^{p_{s} q}\left|\eta_{x_{s}}\right|^{p_{s}} d \mathbf{x} \leqslant C_{6} \int_{\Omega}|u|^{q p_{s}}\left|\eta_{x_{s}}\right|^{p_{s}} d \mathbf{x} .
\end{aligned}
$$

It follows from Lemma 3 for $q_{\alpha}=p_{s}(q-1), \alpha=1,2, \ldots, n$, that

$$
Q=\left(n+p_{s}(q-1) \sum_{\alpha=1}^{n} 1 / p_{\alpha}\right)\left(\sum_{\alpha=1}^{n} 1 / p_{\alpha}-1\right)^{-1}=P+p_{s}(q-1) K
$$

Then (18) and (24) yield

$$
\left(\int_{\Omega}\left|\eta^{p_{s} / p_{1}} u\right|^{P+p_{s}(q-1) K} d \mathbf{x}\right)^{1 / K} \leqslant C_{7} \int_{\Omega}|u|^{q p_{s}}\left|\eta_{x_{s}}\right|^{p_{s}} d \mathbf{x} .
$$

Let $h=p_{s}(q-1)+\theta, \quad \tau=P-K \theta=p_{s}-\theta$, where $\theta=\left(P-p_{s}\right) /(K-1)$. Then $\tau+K h=P+K p_{s}(q-1), \tau+h=p_{s} q$. In view of (13), $K>1$, it follows from condition (7) that $\theta>0$.

We let $\widehat{\rho}+\widehat{\sigma}=\widehat{\rho}_{\nu}=\left(1+2^{-\nu}\right) R, \widehat{\rho}=\widehat{\rho}_{\nu+1}=\left(1+2^{-\nu-1}\right) R, \bar{\rho}-\bar{\sigma}=\bar{\rho}_{\nu}=\left(1-2^{-\nu-1}\right) \varepsilon R$, $\bar{\rho}=\bar{\rho}_{\nu+1}=\left(1-2^{-\nu-2}\right) \varepsilon R, \widehat{\sigma}=R 2^{-\nu-1}, \bar{\sigma}=\varepsilon R 2^{-\nu-2}$. 
By (25) we obtain

$$
\left(\int_{\Omega_{\bar{\rho}}^{\hat{\rho}}}|u|^{\tau+K h} d \mathbf{x}\right)^{1 /(K h)} \leqslant \frac{C_{7}^{1 / h}}{(\min (\bar{\sigma}, \widehat{\sigma}))^{p_{s} / h}}\left(\int_{\Omega_{\bar{\rho}+\widehat{\sigma}}^{\hat{\rho}}}|u|^{\tau+h} d \mathbf{x}\right)^{1 / h} .
$$

We let $h=\theta K^{\nu}, \nu=0,1,2, \ldots$, then

$$
\left(\int_{\Omega_{\bar{\rho}_{\nu+1}}^{\hat{\rho}_{\nu+1}}}|u|^{\tau+\theta K^{\nu+1}} d \mathbf{x}\right)^{1 /\left(\theta K^{\nu+1}\right)} \leqslant \frac{C_{8}^{1 /\left(\theta K^{\nu}\right)} 2^{p_{s}(\nu+1) /\left(K^{\nu} \theta\right)}}{(\varepsilon R)^{p_{s} /\left(K^{\nu} \theta\right)}}\left(\int_{\Omega \widehat{\rho}_{\bar{\rho}}}|u|^{\tau+\theta K^{\nu}} d \mathbf{x}\right)^{1 /\left(\theta K^{\nu}\right)} .
$$

Denoting

$$
\Theta_{\nu}=\left(\int_{\Omega_{\bar{\rho}_{\nu}}^{\widehat{\rho}_{\nu}}}|u|^{\tau+\theta K^{\nu}} d \mathbf{x}\right)^{1 /\left(\theta K^{\nu}\right)}
$$

we get the inequality

$$
\Theta_{\nu+1} \leqslant \frac{C_{8}^{1 /\left(K^{\nu} \theta\right)} 2^{p_{s}(\nu+1) /\left(K^{\nu} \theta\right)}}{(\varepsilon R)^{p_{s} /\left(K^{\nu} \theta\right)}} \Theta_{\nu}, \quad \nu=0,1,2, \ldots
$$

For $\nu=0$ we have $h=\theta, q=1$ and

$$
\Theta_{1} \leqslant \frac{C_{8}^{1 / \theta} 2^{p_{s} / \theta}}{(\varepsilon R)^{p_{s} / \theta}} \Theta_{0}
$$

Hence,

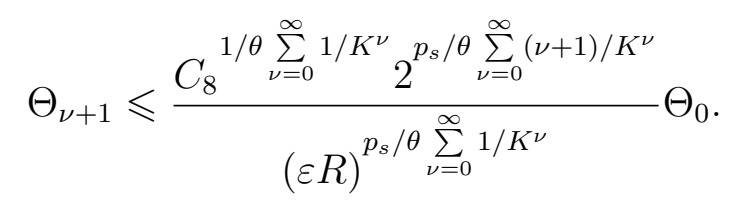

Passing to the limit $\nu \rightarrow \infty$, we obtain

$$
\sup _{\Omega_{\varepsilon R}^{R}}|u(\mathbf{x})| \leqslant \frac{C_{9}}{(\varepsilon R)^{p_{s} K /(\theta(K-1))}}\left(\int_{\Omega_{\varepsilon R / 2}^{2 R}}|u(\mathbf{x})|^{p_{s}} d \mathbf{x}\right)^{1 / \theta} .
$$

In accordance with Corollary 1 and employing (16), we have

$$
\left(\int_{\Omega_{\varepsilon R / 2}^{2 R}}|u|^{p_{s}} d \mathbf{x}\right)^{1 / \theta} \leqslant C_{10} R^{p_{s} / \theta}\left(\int_{\Omega}\left|u_{x_{s}}\right|^{p_{s}} d \mathbf{x}\right)^{1 / \theta} \leqslant C_{11} R^{p_{s} / \theta} .
$$

Combining (26), (27), we finally get

$$
\sup _{\Omega_{\varepsilon R}^{R}}|u| \leqslant C_{12} \frac{R^{p_{s} / \theta}}{(\varepsilon R)^{p_{s} K /(\theta(K-1))}}=\frac{C_{12}}{R^{p_{s} /(\theta(K-1))} \varepsilon^{p_{s} K /(\theta(K-1))}}=\frac{C_{12}}{\left(R \varepsilon^{K}\right)^{M}}
$$

that implies estimate (8). 
Corollary 1. The generalized solution $u(\mathbf{x})$ to problem (1), (2) with functions $\Phi_{\alpha}$, $\alpha=0,1,2, \ldots, n$, obeying (6), under the hypothesis of Theorem 1 satisfies the estimate

$$
\sup _{\Omega_{2} R_{0}}|u| \leqslant \widehat{C}
$$

where $\widehat{C}$ is a constant independent of $p_{\alpha}, n, \bar{a}, \widehat{a},\left\|\Phi_{\alpha}\right\|_{p_{\alpha} /\left(p_{\alpha}-1\right)}, R_{0}$.

Proof. In 28) we let $\varepsilon=1 / 2, R=r_{k}=2^{k+1} R_{0}, k=1,2, .$. , that lead us to the inequalities

$$
\sup _{\Omega_{r_{k} / 2}^{r_{k}}}|u| \leqslant C_{13} 2^{(K-k) M} \leqslant \widehat{C}, \quad k=1,2, \ldots,
$$

implying (29).

Proof of Theorem 2. The proof is similar to that of Theorem 1. However, there are some differences in construction of cut-off functions and estimates related with $\Phi_{\alpha}, \alpha=\overline{1, n}$, and thus we provide it in all detail.

Let $\eta\left(x_{s}\right)$ be non-negative Lipschitz function with a support in $(-\infty, \rho+\sigma), \rho+\sigma \leqslant 2 R_{1}$, such that

$$
\eta\left(x_{s}\right)= \begin{cases}1, & x_{s} \in(-\infty, \rho], \\ 0, & x_{s} \in[\rho+\sigma,+\infty), \\ \text { linear, } & x_{s} \in(\rho, \rho+\sigma) .\end{cases}
$$

We let $v(\mathbf{x})=\eta^{p_{s}} G(u) \in \stackrel{\circ}{H_{\mathbf{p}}^{1}}(\Omega), \chi=1$. Employing $(19)$, we find

$$
\begin{aligned}
L(u, v)= & \sum_{\alpha=1}^{n}\left(a_{\alpha}(\mathbf{x}, \nabla u)+\Phi_{\alpha}\right)\left(p_{s} \eta^{p_{s}-1} G(u) \eta_{x_{\alpha}}+\eta^{p_{s}} G^{\prime}(u) u_{x_{\alpha}}\right) \\
\geqslant & \eta^{p_{s}} F^{\prime}(\bar{u})^{p_{s}} \sum_{\alpha=1}^{n} a_{\alpha}(\mathbf{x}, \nabla u) u_{x_{\alpha}}-p_{s} \eta^{p_{s}} F^{\prime}(\bar{u})^{p_{s}} \sum_{\alpha=1}^{n}\left|\Phi_{\alpha}\right|\left|\bar{u}_{x_{\alpha}}\right| \\
& \quad-p_{s} \eta^{p_{s}-1}\left|\eta_{x_{s}}\right| F(\bar{u}) F^{\prime}(\bar{u})^{p_{s}-1}\left|a_{s}(\mathbf{x}, \nabla u)\right|-p_{s} \eta^{p_{s}-1}\left|\eta_{x_{s}}\right| F(\bar{u}) F^{\prime}(\bar{u})^{p_{s}-1}\left|\Phi_{s}\right| .
\end{aligned}
$$

Employing conditions (3)-(5), we obtain

$$
\begin{aligned}
L(u, v) \geqslant & \bar{a} \eta^{p_{s}} F^{\prime}(\bar{u})^{p_{s}} \sum_{\alpha=1}^{n}\left|\bar{u}_{x_{\alpha}}\right|^{p_{\alpha}}-\widehat{a} p_{s} \eta^{p_{s}-1} F(\bar{u}) F^{\prime}(\bar{u})^{p_{s}-1}\left|\eta_{x_{s}}\right|\left|\bar{u}_{x_{s}}\right|^{p_{s}-1} \\
& -p_{s} \eta^{p_{s}} F^{\prime}(\bar{u})^{p_{s}} \sum_{\alpha=1}^{n}\left|\Phi_{\alpha}\right|\left|\bar{u}_{x_{\alpha}}\right|-p_{s} \eta^{p_{s}-1} F(\bar{u}) F^{\prime}(\bar{u})^{p_{s}-1}\left|\eta_{x_{s}}\right|\left|\Phi_{s}\right| .
\end{aligned}
$$

We integrate $(30)$ over $\mathbf{x} \in \Omega$ and in view of $(15)$, we obtain

$$
\begin{aligned}
\int_{\Omega} \eta^{p_{s}} F^{\prime}(\bar{u})^{p_{s}} \sum_{\alpha=1}^{n}\left|\bar{u}_{x_{\alpha}}\right|^{p_{\alpha}} d \mathbf{x} \leqslant & C_{1} \int_{\Omega} F(\bar{u}) F^{\prime}(\bar{u})^{p_{s}-1} \eta^{p_{s}-1}\left|\eta_{x_{s}}\right|\left(\left|\bar{u}_{x_{s}}\right|^{p_{s}-1}+\left|\Phi_{s}\right|\right) d \mathbf{x} \\
& +C_{1} \int_{\Omega} \eta^{p_{s}} F^{\prime}(\bar{u})^{p_{s}} \sum_{\alpha=1}^{n}\left|\Phi_{\alpha}\right|\left|\bar{u}_{x_{\alpha}}\right| d \mathbf{x} .
\end{aligned}
$$

Applying Young inequality, we get

$$
\begin{aligned}
\int_{\Omega} \eta^{p_{s}} F^{\prime}(\bar{u})^{p_{s}} & \sum_{\alpha=1}^{n}\left|\bar{u}_{x_{\alpha}}\right|^{p_{\alpha}} d \mathbf{x} \leqslant \frac{1}{2} \sum_{\alpha=1}^{n} \int_{\Omega} \eta^{p_{s}} F^{\prime}(\bar{u})^{p_{s}}\left|\bar{u}_{x_{\alpha}}\right|^{p_{\alpha}} d \mathbf{x} \\
& +C_{2} \int_{\Omega} F(\bar{u})^{p_{s}}\left|\eta_{x_{s}}\right|^{p_{s}} d \mathbf{x}+C_{2} \sum_{\alpha=1}^{n} \int_{\Omega} \eta^{p_{s}} F^{\prime}(\bar{u})^{p_{s}}\left|\Phi_{\alpha}\right|^{p_{\alpha} /\left(p_{\alpha}-1\right)} d \mathbf{x} .
\end{aligned}
$$


Taking into consideration (20), we obtain

$$
\sum_{\alpha=1}^{n} \int_{\Omega} \eta^{p_{s}} F^{\prime}(\bar{u})^{p_{s}}\left|\bar{u}_{x_{\alpha}}\right|^{p_{\alpha}} d \mathbf{x} \leqslant C_{3} \int_{\Omega} \bar{u}^{q p_{s}}\left|\eta_{x_{s}}\right|^{p_{s}} d \mathbf{x}+C_{3} \sum_{\alpha=1}^{n} \int_{\Omega}\left|\Phi_{\alpha}\right|^{p_{\alpha} /\left(p_{\alpha}-1\right)} \eta^{p_{s}} \bar{u}^{p_{s}(q-1)} d \mathbf{x} .
$$

Assume that the right hand side of (31) is finite. We let $\mu$ tend to infinity in the left hand side of (31) and apply Fatou lemma

$$
\int_{\Omega^{\rho}} \bar{u}^{p_{s}(q-1)} \sum_{\alpha=1}^{n}\left|\bar{u}_{x_{\alpha}}\right|^{p_{\alpha}} d \mathbf{x} \leqslant \frac{C_{3}}{q^{p_{s}}}\left(\frac{1}{\sigma^{p_{s}}} \int_{\Omega^{\rho+\sigma}} \bar{u}^{q p_{s}} d \mathbf{x}+\sum_{\alpha=1}^{n} \int_{\Omega^{\rho+\sigma}}\left|\Phi_{\alpha}\right|^{p_{\alpha} /\left(p_{\alpha}-1\right)} \bar{u}^{p_{s}(q-1)} d \mathbf{x}\right) .
$$

Applying Hölder inequality and employing (9), we arrive at the inequalities

$$
\begin{aligned}
& \sum_{\alpha=1}^{n} \int_{\Omega^{\rho}} \bar{u}^{p_{s}(q-1)}\left|\bar{u}_{x_{\alpha}}\right|^{p_{\alpha}} d \mathbf{x} \leqslant \frac{C_{3}}{q^{p_{s}}}\left(\frac{1}{\sigma^{p_{s}}}\left(\int_{\Omega^{\rho+\sigma}} \bar{u}^{q p_{s} l} d \mathbf{x}\right)^{1 / l}\left(\operatorname{mes} \Omega^{2 R_{1}}\right)^{(l-1) / l}\right. \\
& \left.\quad+\sum_{\alpha=1}^{n}\left(\int_{\Omega^{\rho+\sigma}}\left|\Phi_{\alpha}\right|^{k_{\alpha}} d \mathbf{x}\right)^{(l-1) / l}\left(\int_{\Omega^{\rho+\sigma}} \bar{u}^{p_{s}(q-1) l} d \mathbf{x}\right)^{1 / l}\right) \leqslant C_{4}\left(1+\frac{1}{\sigma^{p_{s}}}\right)\left(\int_{\Omega^{\rho+\sigma}} \bar{u}^{q p_{s} l} d \mathbf{x}\right)^{1 / l} .
\end{aligned}
$$

Taking into consideration Remark, we apply Lemma 3 for $D=\Omega^{\rho}$ and function $u \in \stackrel{\circ}{H} \mathbf{p}(\Omega)$. Thus, employing (18), we obtain

$$
\left(\int_{\Omega^{\rho}}|u|^{P+p_{s}(q-1) K} d \mathbf{x}\right)^{1 / K} \leqslant C_{5}\left(1+\frac{1}{\sigma^{p_{s}}}\right)\left(\int_{\Omega^{\rho+\sigma}} \bar{u}^{q p_{s} l} d \mathbf{x}\right)^{1 / l} .
$$

Due to (10), $K>l$. Employing then (32), we get the following chain of inequalities

$$
\begin{aligned}
\int_{\Omega^{\rho}} \bar{u}^{P+p_{s}(q-1) K} d \mathbf{x} & \leqslant C_{6} \int_{\Omega^{\rho}}|u|^{P+p_{s}(q-1) K} d \mathbf{x}+C_{6} \operatorname{mes} \Omega^{\rho} \\
& \leqslant C_{7}\left(1+\frac{1}{\sigma^{p_{s}}}\right)^{K}\left(\int_{\Omega^{\rho+\sigma}} \bar{u}^{q p_{s} l} d \mathbf{x}\right)^{K / l}+C_{6} \int_{\Omega^{\rho+\sigma}} \bar{u}^{q p_{s} l} d \mathbf{x} \\
& \leqslant C_{8}\left(1+\frac{1}{\sigma^{p_{s}}}\right)^{K}\left(\int_{\Omega^{\rho+\sigma}} \bar{u}^{q p_{s} l} d \mathbf{x}\right)^{K / l} .
\end{aligned}
$$

We let $\rho+\sigma=\rho_{\nu}=\left(1+2^{-\nu}\right) R_{1}, \rho=\rho_{\nu+1}=\left(1+2^{-\nu-1}\right) R_{1}, \sigma=R_{1} 2^{-\nu-1}, h=l p_{s}(q-1)+l \theta$, $\tau=P-K \theta=l\left(p_{s}-\theta\right)$, where $\theta=\left(P-l p_{s}\right) /(K-l)$. Then $\tau+h m=P+K p_{s}(q-1), m=K / l$, $\tau+h=l p_{s} q$. It follows from (10) that $\theta>0$.

By (33) we get

$$
\left(\int_{\Omega^{\rho}}|u|^{\tau+m h} d \mathbf{x}\right)^{1 /(m h)} \leqslant \frac{C_{9}^{1 / h}}{\sigma^{p_{s} l / h}}\left(\int_{\Omega^{\rho+\sigma}}|u|^{\tau+h} d \mathbf{x}\right)^{1 / h}
$$

Leth $=l \theta m^{\nu}, \nu=0,1,2, \ldots$, then

$$
\left(\int_{\Omega^{\rho_{\nu+1}}}|u|^{\tau+l \theta m^{\nu+1}} d \mathbf{x}\right)^{1 /\left(l \theta m^{\nu+1}\right)} \leqslant \frac{C_{9}^{1 /\left(l \theta m^{\nu}\right)} 2^{p_{s}(\nu+1) /\left(m^{\nu} \theta\right)}}{R_{1}^{p_{s} /\left(\theta m^{\nu}\right)}}\left(\int_{\Omega^{\rho \nu}}|u|^{\tau+l \theta m^{\nu}} d \mathbf{x}\right)^{1 /\left(l \theta m^{\nu}\right)} .
$$


Denoting

$$
\Theta_{\nu}=\left(\int_{\Omega^{\rho_{\nu}}}|u|^{\tau+l \theta m^{\nu}} d \mathbf{x}\right)^{1 /\left(l \theta m^{\nu}\right)}
$$

we arrive at the inequality

$$
\Theta_{\nu+1} \leqslant \frac{C_{9}{ }^{1 /\left(l \theta m^{\nu}\right)} 2^{p_{s}(\nu+1) /\left(m^{\nu} \theta\right)}}{R_{1}^{p_{s} /\left(\theta m^{\nu}\right)}} \Theta_{\nu}, \quad \nu=0,1,2, \ldots
$$

This inequality yields

$$
\Theta_{\nu+1} \leqslant \frac{C_{9}^{1 /(l \theta)} \sum_{\nu=0}^{\infty} 1 / m^{\nu} 2^{p_{s} / \theta \sum_{\nu=0}^{\infty}(\nu+1) / m^{\nu}}}{R_{1} / \theta \sum_{\nu=0}^{\infty} 1 / m^{\nu}} \Theta_{0}, \quad \nu=0,1,2, \ldots
$$

Passing to the limit $\nu \rightarrow \infty$, we obtain

$$
\sup _{\Omega^{R_{1}}} \bar{u}(\mathbf{x}) \leqslant C_{10}\left(\int_{2^{2 R_{1}}} \bar{u}(\mathbf{x})^{p_{s} l} d \mathbf{x}\right)^{1 /(\theta l)}
$$

By (10), (14), (16) we get

$$
\int_{\Omega^{2 R_{1}}} \bar{u}^{p_{s} l} d \mathbf{x} \leqslant \int_{\Omega^{2 R_{1}}} \bar{u}^{P} d \mathbf{x} \leqslant C_{11} \text { mes } \Omega^{2 R_{1}}+C_{11} \int_{\Omega}|u|^{P} d \mathbf{x} \leqslant C_{12} .
$$

Combining (34), (35), we finally obtain 12 .

\section{BIBLIOGRAPHY}

1. I.M. Kolodii. On boundedness of generalized solution to elliptic differential equations // Vestnik MGU. 5:5, 45-52 (1970). (in Russian).

2. L.M. Kozhevnikova, A.A. Khadzhi. Solutions of anisotropic elliptic equations in unbounded domains // Vestn. Samar. Gos. Tekhn. Univ. Ser. Fiz.-Mat. Nauki. 30:1, 90-96 (2013). (in Russian).

3. Lu Ven-Tuan. On embedding theorems for spaces of functions with partial derivatives summable with various powers // Vestnik LGU. 7, 23-27 (1961). (in Russian).

4. S.N. Kruzhkov. Boundary value problems for degenerate second order elliptic equations // Matem. Sbornik. 77:3, 229-334 (1968). [Math. USSR-Sb. 6:3, 275-307 (1968).]

5. L.M. Kozhevnikova, A.A. Leontiev. Estimates of solutions of anisotropic doubly nonlinear parabolic equation // Ufimskij Matem Zhurn. 3:4, 64-85 (2011). [Ufa Math. J. 3:4, 62-83 (2011).]

6. L. Nirenberg. On elliptic partial differential equations // Ann. Scuola norm. super. Pisa. 13:2 115-162 (1959).

7. Yu.A. Dubinskii. Some integral inequalities and the solvability of degenerate quasi-linear elliptic systems of differential equations // Matem. Sbornik. 64(106):3, 458-480 (1964). (in Russian).

8. V.S. Klimov. On imbedding theorems for anisotropic classes of functions // Matem. Sbornik. 127(169):2(6), 198-208 (1985). [Math. USSR-Sb. 55: 1, 195-205 (1986).]

9. J. Moser. A new proof of de Giorgi's theorem concerning the regularity problem for elliptic differential equations // Communs Pure and Appl. Math. 1960. 3. P. 457-468.

10. S.N. Kruzhkov. A priori estimates for generalized solutions of second-order elliptic and parabolic equations // Dokl. Akad. Nauk SSSR. 150:3, 470-473 (1963). [Sov. Math. Dokl. 4:3, 757-761 (1963).]

11. J. Serrin. Local behavior of solutions of quasilinear equatons // Acta math. 111:1, 247-302 (1964). 
Larisa Mikhailovna Kozhevnikova,

Sterlitamak Branch of Bashkir State University,

Lenin av., 37,

453103, Sterlitamak, Russia

E-mail: kosul@mail.ru

Anna Alexandrovna Khadzhi,

Sterlitamak Branch of Bashkir State University,

Lenin av., 37,

453103, Sterlitamak, Russia

E-mail: anna_5955@mail.ru 